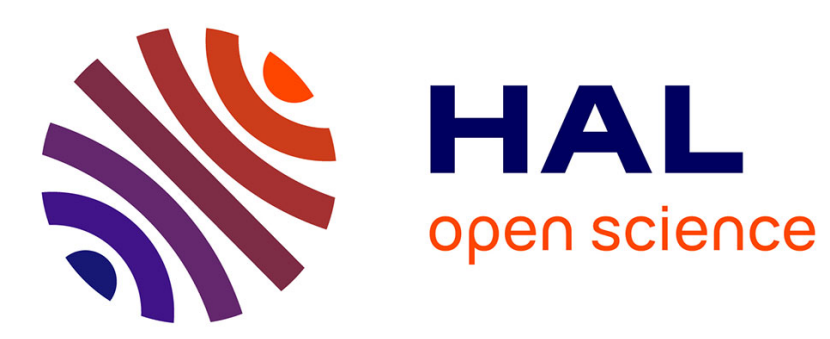

\title{
Roland Barthes, lo fílmico versus el cine. El sentido suspendido como definición de la modernidad cinematográfica
}

\author{
Lourdes Monterrubio Ibáñez
}

\section{- To cite this version:}

Lourdes Monterrubio Ibáñez. Roland Barthes, lo fílmico versus el cine. El sentido suspendido como definición de la modernidad cinematográfica. Escritura e imagen, 2021, 17, pp.197-216. 10.5209/esim.78941 . hal-03475932

\section{HAL Id: hal-03475932 \\ https://hal.science/hal-03475932}

Submitted on 11 Dec 2021

HAL is a multi-disciplinary open access archive for the deposit and dissemination of scientific research documents, whether they are published or not. The documents may come from teaching and research institutions in France or abroad, or from public or private research centers.
L'archive ouverte pluridisciplinaire HAL, est destinée au dépôt et à la diffusion de documents scientifiques de niveau recherche, publiés ou non, émanant des établissements d'enseignement et de recherche français ou étrangers, des laboratoires publics ou privés. 


\title{
Escritura e Imagen
}

ISSN: $1885-5687$

\section{Roland Barthes, lo fílmico versus el cine. El sentido suspendido como definición de la modernidad cinematográfica}

\author{
Lourdes Monterrubio Ibáñez
}

Recibido: 01-08-2021 / Aceptado: 03-11-2021

Resumen. El presente artículo pretende analizar los textos de Roland Barthes sobre la práctica cinematográfica. Su resistencia al cine, vinculada a su escritura autobiográfica, se revela como la imposibilidad de fetichizar la imagen en movimiento, que debe entonces ser reducida a lo fotográfico, definiendo así un sentido obtuso que opone lo fílmico al cine. Sin embargo, a través de la concepción del sentido suspendido, Barthes detectó el surgimiento de una modernidad cinematográfica que era capaz, precisamente, de superar ese límite; a saber, destruir la imagen-acción y su temporalidad sin detener el movimiento. La elección de obras de Buñuel, Bergman, Resnais, Pasolini y Antonioni configura una concepción del sentido suspendido vinculada a la suspensión de la temporalidad y el extrañamiento que prefiguran la imagen-tiempo deleuziana. Barthes halla en la modernidad cinematográfica el punto ciego de su capacidad analítica, la de una imagen que suspende el sentido sin detener su movimiento.

Palabras clave: Roland Barthes; sentido suspendido; modernidad cinematográfica, imagen-tiempo

\section{[en] Roland Barthes, the filmic versus cinema. The suspended sense as definition of cinematic modernity}

\begin{abstract}
This article aims to analyse Roland Barthes' texts on film practice. His resistance to cinema, linked to his autobiographical writing, is revealed as the impossibility of fetishizing the moving image, which must then be reduced to the photographic, thus defining the obtuse sense. However, through the conception of suspended sense, Barthes detected the emergence of cinematic modernity that, precisely, was capable of overcoming this limit; that is to say, to destroy the action-image and its temporality without stopping its movement. The choice of works by Buñuel, Bergman, Resnais, Pasolini and Antonioni establishes a conception of the suspended sense linked to the suspension of temporality and estrangement that prefigure the Deleuzian time-image. Barthes finds in modern cinema the blind spot of his analytical capacity, that of an image that suspends sense without stopping its movement.
\end{abstract}

Keywords: Roland Barthes; suspended sense; cinematic modernity; time-image.

Sumario: 1. Introducción; 2. Desmitificar el cine; 3. Hacia una semiótica del cine; 4. El sentido obtuso; 5. Michelangelo Antonioni; 6. Conclusiones; 7. Referencias bibliográficas.

Cómo citar: Monterrubio Ibáñez, L. (2021) "Roland Barthes, lo fílmico versus el cine. El sentido suspendido como definición de la modernidad cinematográfica”, en Escritura e Imagen 17, 197-216. 


\section{Introducción}

El objetivo de este artículo es el de abordar el estudio de los textos barthesianos sobre la práctica cinematográfica, dejando a un lado los dedicados a la imagen fotográfica. Es decir, hacer un ejercicio restrictivo con el propósito de alcanzar algunas conclusiones sobre la relación de Roland Barthes con el cine, tomando en consideración los últimos estudios publicados sobre la cuestión ${ }^{1}$. Para realizar este análisis, es preciso partir de una realidad indiscutible, enunciada por el autor en diferentes ocasiones, su resistencia al cine. En La Chambre claire afirma: "Je décrétai que j'aimais la photo contre le cinéma, dont je n'arrivais pas cependant à les séparer"'. Y en una de las entrevistas recogidas en en Le Grain de la voix añade:

Je veux dire aussi que, si j'ai choisi la photographie, c'est un peu contre le cinéma. J'ai constaté que j'avais un rapport positif à la photographie, j'aime voir des photographies, et, par contre un rapport difficile et résistant au cinéma [...] je place la photographie paradoxalement au-dessus du cinéma, dans mon petit panthéon personnel. ${ }^{3}$

Es a partir de esta resistencia desde donde describiremos la oposición que enunciamos en el titulo, lo fílmico versus el cine, que en nuestra opinión es la paradójica esencia de los escritos de Barthes sobre el arte cinematográfico. Sobre esta resistencia frente al cine, Wattsnchre, alineándose con los autores que ven en la trayectoria de Barthes una permanente dialéctica entre dos posicionamientos, expone:

La résistance est une sorte de compromis entre la fascination et la répulsion, ou plutôt une alternance entre enchantement et critique, une façon de simultanément se délecter et se détourner de l'image. Tout au long de sa vie, Barthes aura écrit sur le cinéma comme pour tenter de comprendre et de « travailler » cette résistance qui était la sienne. ${ }^{4}$

Y describe esa resistencia como: "un désir à la fois de prendre du plaisir et de démystifier un système esthétique que le fascine et qu'il considère néanmoins comme

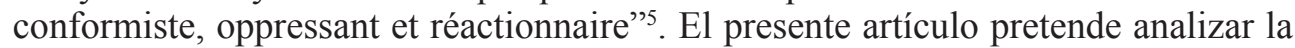
evolución de esa contradicción. Frente a la realidad fílmica de casi cuatro décadas, Barthes sostuvo dos posicionamientos radicalmente opuestos, dos posiciones casi antagónicas sobre las que algunos autores argumentan una evolución basada en una dialéctica de esa resistencia frente a lo fílmico, y sobre las que otros interpretan una suerte de giro radical: "les mille et une contradiction qui parcourent l'œuvre de Barthes, et c'est là sa faiblesse doctrinale [...] Or, sa faiblesse fut aussi sa grandeur"; "première volte-face théorique: une prise de distance vis-à-vis de l'émotion"; "seconde volte-face théorique [...] une prise de distance vis-à-vis de la politique"6".

\footnotetext{
Watts, P., Le cinéma de Roland Barthes. Suivi d'un entretien avec Jacques Rancière, Paris, De l'incidence, 2015; Didi-Huberman, G., Peuples en larmes, peuples en armes. L'oeil de l'Histoire, 6, Paris, Éditions de Minuit, 2016; Ffrench, P., Roland Barthes and Film. Myth, Eroticism and Poetics, London, Bloomsbury Academic, 2020.

Barthes, R., La Chambre claire, Paris, Éditions de l'Étoile, Gallimard, Le Seuil, 1980, p. 13.

Barthes, R., Le Grain de la voix : entretiens, 1962-1980, Paris, Éditions du Seuil, 1981, p. 334.

Watts, P., Le cinéma de Roland Barthes. Suivi d'un entretien avec Jacques Rancière, op. cit., p. 33.

Ibidem, p. 61.

Didi-Huberman, G., Peuples en larmes, peuples en armes. L'oeil de l'Histoire, 6, op. cit., pp. 89, 93, 96.
} 


\section{Desmitificar el cine}

En la primera vertiente de su escritura sobre el cine, desarrollada en los años 40 y 50 y cuyos textos son recogidos en su mayoría en Mythologies (1957), Barthes realiza una crítica marxista y del distanciamiento brechtiano frente al objeto cinematográfico, entendido este último como un producto del capitalismo que es preciso desmitificar. En esta primera etapa, dedicada a esta desmitificación del cine, Barthes comparte con otros autores de la época:

une conception du cinéma comme opium du peuple, comme attraction populaire aux effets nocifs sur les masses [...] Le problème n'était pas de définir le cinéma comme un art, mais de se réapproprier l'art du cinéma en sortant de l'illusion, de le sauver de la mystification du spectacle capitaliste et de ses déterminations sociales et économiques. ${ }^{7}$

De este modo, Barthes se convierte en uno de los representantes de la hermenéutica de la sospecha -utilizando el concepto de Paul Ricœur (1986)- que persigue dar detallada cuenta de la mistificación que transforma la cultura pequeño-burguesa en naturaleza universal. Una crítica ideológica del capitalismo y de la cultura de masas que se esfuerza en desmitificar su presencia en los objetos fílmicos, especialmente de los productos americanos, mediante "the constant denunciation of Hollywood artífice and excess" izquierdas que combate el abuso ideológico del capitalismo. Sobre esta primera vertiente barthesiana, Jacques Rancière afirma:

À mes yeux, toutefois, cela ne définit pas vraiment un intérêt propre de Roland Barthes pour le cinéma comme tel. Les images empruntées au cinéma sont là à côté d'images empruntées à la publicité, aux photographies de reportage, à la presse, ne démontrant pas, à l'époque de Mythologies du moins, un intérêt spécifique de Roland Barthes pour le cinéma. ${ }^{9}$

Recogemos a lo largo del texto las películas abordadas de forma más concreta en los escritos de Barthes, a fin de analizar sus aportaciones. El listado completo de todas las películas citadas por el autor lo realiza Patrick Ffrench en su texto. ${ }^{10}$

\section{Textos}

1943 Les Anges du péché

1953 Pouvoirs de la tragédie antique

1953 Visages et Figures

1954 Au cinémascope

1954 Versailles et ses comptes

\section{Películas}

Les Anges du péché (1943) Robert Bresson

Breif Encounter (1945) David LEan

Umberto D (1952) Vittorio de Sica

The Robe (1953) H. Koster

El acorazado Potemkin (1925)

Sergie M. Eisenstein

Si Versailles m'était conté (1954) Sacha Guitry

Watts, P., Le cinéma de Roland Barthes. Suivi d'un entretien avec Jacques Rancière, op.cit., p. 40.

Watts, P., "Roland Barthes's Cold-War Cinema", SubStance, \#108, 34(3), 2005, p. 21.

Watts, P., Le cinéma de Roland Barthes. Suivi d'un entretien avec Jacques Rancière, op. cit., p. 165.

Ffrench, P., Roland Barthes and Film. Myth, Eroticism and Poetics, op. cit. pp. 276-279. 
Mythologies (1957)

1957 L'acteur d'Harcourt

1957 Les Romains au cinéma

1957 Le Pauvre et le Prolétaire

1957 Iconographie de l'abbé Pierre

1957 Un ouvrier sympathique

1957 Le visage de Garbo

1957 Puissance et désinvolture

1957 Continent perdu

1959 Cinéma droite et gauche
Julius Caesar (1953) Joseph L. Mankiewicz

Modern Times (1936) Charles Chaplin

Les Chiffonniers d'Emmaus (1954)

Boris Simon

On the Waterfront (1954) Elia Kazan

Queen Christina (1933) Rouben Mamoulian

Touchez pas au grisbi (1954) Jacques Becker

Continente perduto (1954) M. Gras, G. Moser

Le Beau Serge (1959) Claude Chabrol

La terra trema (1948) Luccino Visconti

El corto texto dedicado al film de Bresson se limita a halagar el realismo de la obra, que no sucumbe a las pretenciosas aspiraciones de los films religiosos, entre los que cita L'Appel du silence (Léon Poirier, 1936) como ejemplo. La veracidad del film de Bresson descansa principalmente, en opinión de Barthes, en los diálogos de Jean Giradoux. Por el contrario, el film de Guitry encarnaría la necesidad de desmitificación de un cine que traiciona la historia y que idiotiza al espectador: "En somme, le film vise à humilier [...] on trait l'homme comme le chien de Pavlov [...] ce n'est pas un film pour hommes, c'est un film pour bêtes [...] la pavlovisation du spectateur se fait avec le concours de l'État, avec son château, sinon avec ses millions"11. Esta crítica se halla en total sintonía con el texto de François Truffaut, "Une certaine tendance du cinéma français" (1954), publicado solo unos meses antes. Las diferencias entre los cines que representan ambas obras quedan igualmente ilustradas en "Au cinémascope", donde Barthes se pregunta si las para él virtudes de este formato se pondrán al servicio de uno u otro: "Ceci devrait être, à proprement parler, l'espace de l'Histoire et, techniquement, la dimension épique est née [...] voilà qui est maintenant possible; le balcon de l'Histoire est prêt. Reste à savoir ce qu'on nous y montrera ; si ce sera Potemkine ou La Tunique, Odessa ou SaintSulpice, l'Histoire ou la Mythologie"12.

En "L'acteur d'Harcourt" el autor hace la misma distinción en relación a la función social de los actores. Entre las fotografías de los estudios Harcourt: "Par un scrupule d'illusion bien propre à une époque et à une classe sociale trop faibles à la fois pour la raison pure et le mythe puissant, la foule [...] déclare que ces faces irréelles sont celles-là mêmes de la ville et se donne ainsi la bonne conscience rationaliste de supposer un homme derrière l'acteur"; y las tomadas por Thérèse Le Prat o Agnès Varda: "elles laissent toujours à l'acteur son visage d'incarnation et l'enferment franchement, avec une humilité exemplaire, dans sa fonction sociale, qui est de " représenter », et non de mentir" ${ }^{\prime 3}$. Se trata de la misma distinción entre los actores del cine clásico y los perteneciente al moderno que hace en "Le visage de Garbo"; entre el rostro de la actriz en Queen Christina y el de Katherine Hepburn. Mientras que en el primero remite al mito: "C'est sans doute un admirable visage-objet

Barthes, R., Euvres Complètes Tome I, 1942-1961, Paris, Éditions du Seuil, 2002, p. 402.

Ibidem, p. 380.

Ibidem, pp. 577-578. 
[...] un archétype du visage humain", "Le visage de Garbo représente ce moment fragile, où le cinéma va extraire une beauté existentielle d'une beauté essentielle, où l'archétype va s'infléchir vers la fascination des figures périssables"; el segundo materializa la modernidad actual: "Comme langage, la singularité de Garbo était d'ordre conceptuel, celle d'Audrey Hepburn est d'ordre substantiel. Le visage de Garbo est Idée, celui de Hepburn est Événement". ${ }^{14}$ Como indica Ffrench: "The distinction Barthes makes in 'The Face of Garbo,' between the tyrannical beauty of the face of Garbo, as Idea or Concept, and the face of Audrey Hepburn, as Event, points to a cinematographic physiognomy finally freed from the demands of ritual, and responsive to the singularity of what occurs" 15 .

Desde diferentes perspectivas, Barthes se reitera continuamente en la misma disyuntiva entre las experiencias clásicas y las innovaciones de la modernidad cinematográfica. En el caso de "Les Romains au cinéma" establece la diferencia entre el signo extremo "franchement intellectuel" o "profondément enraciné, inventé en quelque sorte à chaque fois"; y el signo intermedio, como en Julius Caesar, que "dénonce un spectacle dégradé, qui craint autant la vérité naïve que l'artifice total [...] entre le signe intellectuel et le signe viscéral, cet art dispose hypocritement un signe bâtard, à la fois elliptique et prétentieux, qu'il baptise du nom pompeux de « naturel »" ${ }^{16}$ Barthes determina la pertenencia de las obras a uno u otro espacio, con apreciaciones muy relevantes desde el punto de vista del análisis cultural, pero que nunca se adentra en los territorios del lenguaje cinematográfico. Respecto a Modern Times, expone con lucidez los logros de la obra en su representación de un proletario ajeno aún a la lucha revolucionaria:

Aucune œuvre socialiste n'est encore arrivée à exprimer la condition humiliée du travailleur avec autant de violence et de générosité. Seul Brecht, peut-être, a entrevu la nécessite pour l'art socialiste de prendre toujours l'homme à la veille de la Révolution, c'est-à-dire l'homme seul, encore aveugle, sur le point d'être ouvert à la lumière révolutionnaire par l'excès «naturel » de ses malheurs. ${ }^{17}$

La película de Kazan, sin embargo, encarna a la perfección la mistificación que Barthes denuncia: "il s'agit encore une fois de cette vaccine de la vérité dont j'ai indiqué le mécanisme tout moderne à propos d'autres films américains: on dérive sur un petit groupe de gangsters la fonction d'exploitation du grand patronat, et par ce petit mal confessé [...] on détourne du mal réel, on évite de le nommer, on l'exorcise"18, de la misma forma que lo hace respecto del gesto del gánster en Touchez pas au grisbi o del mito exótico en Continente perduto. Se trata, por tanto, de analizar y denunciar la función social del cine, en tanto que herramienta ideológica del capitalismo, como el propio Barthes evidencia dos años más tarde en "Cinéma droite et gauche":

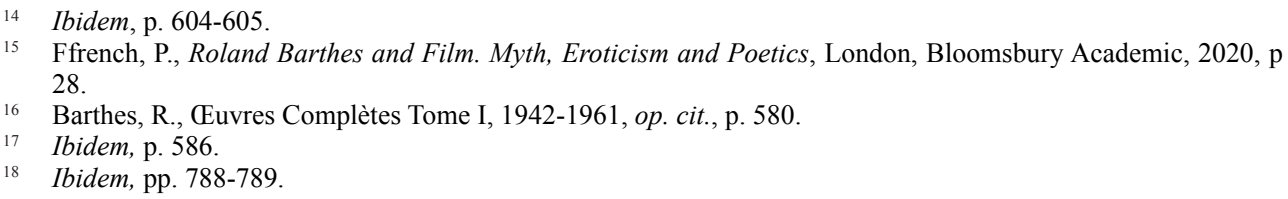


le talent est à droite et la vérité à gauche [...] que la vérité est dans le style, la concession dans le fond, et que par un paradoxe de structure l'être du récit n'est qu'un attribut de sa forme ; d'où un divorce général entre une vérité des signes, toute une façon moderne de voir justement la surface du monde, et une imposture des arguments et des rôles, tirés négligemment du folklore bourgeois le plus grossier [...] on pourrait même dire qu'actuellement toute notre avant-garde vit de cette contradiction: des signes vrais, un sens faux. ${ }^{19}$

Así Barthes sitúa Le Beau Serge en "un art de droit, toujours intéressé par le discontinu des malheurs humains, jamais par leur liaison", que define como "cette fascination de l'immobilité, qui fait que l'on décrit des résultats sans jamais s'interroger, je ne dis pas sur les causes (un art ne saurait être déterministe) mais sur les fonctions [...] En somme, ce que l'anecdote permet à Chabrol d'esquiver, c'est le réel"20; y que nuevamente lo opone a otra obra, La terra trema. La tarea de desmitificación de Barthes no pasa entonces por el análisis del lenguaje cinematográfico, sino por el de la representación que este genera: "Again Barthes' critique focuses on an aesthetic which does not go far enough, which is content to remain at the surface level of signs, without exhausting them, without pushing further below the surface and allowing for a work of thought" 21 .

\section{Hacia una semiótica del cine}

En la década de los sesenta Barthes presenta una aproximación de lo que debiera ser un análisis semiótico del objeto fílmico, expuesto en cuatro escritos clave (dos artículos y dos entrevistas), que anuncian la gran sintagmática de Christian Metz. Textos donde señala las líneas de investigación de una semiótica cinematográfica que en ningún caso él desarrollará:

1960 Le problème de la signification au cinéma

Signifiant - signifié

Le Beau Serge (1959) Claude Chabrol

Bob le flambeur (1956) Jean-Pierre Melville

1960 Les « unités traumatiques » au cinéma (con Gilbert Cohen-Séat)

1961 Les Inconnus de la terre (1961) Mario Ruspoli

Chronique d'un été (1961) Edgar Morin y Jean Rouch

1963

Sur le cinéma (entrevista con Delahaye y Rivette, Cahiers du cinéma)

Le sens suspendu $=$ signifiance (Lacan)

El ángel exterminador (1962) Luis Buñuel

L'Immortelle (1963) Alain Robbe-Grillet

$9 \quad$ Ibidem, p. 787.

Ibidem, pp. 788-789.

Ffrench, P., Roland Barthes and Film. Myth, Eroticism and Poetics, op. cit., p. 47. 
1964

Sémiologie et cinéma (entrevista con Pilard y Tardy, Image et Son)

Retórica - signo - eje paradigmático

Estructuralismo - significancia - eje sintagmático

The Silence (1963) Ingmar Bergman

L'Homme de Rio (1964) Philippe de Broca

1966 Principi e scopi dell'analisi strutturale (conferencia Festival de Cine de Pessaro)

\section{L'Année dernière à Marienbad (1961) Alain Resnais}

En "Le problème de la signification au cinéma" Barthes describe el marco de una semiología de la imagen fílmica, presentando las instancias del emisor/autor y receptor/espectador del mensaje fílmico para a continuación definir los conceptos de significante (heterogéneo, polivalente y combinatorio) y significado y su relación analógica (analogon). El autor ilustra lo expuesto mediante dos tablas de ejemplos signifiant - signifié analizando el film Le Beau Serge. Se nos revela entonces una semiología del interior de la imagen, del vestuario y el decorado, de la dirección artística, que en ningún caso hace referencia al dispositivo cinematográfico, a la expresión formal de la imagen: posición de cámara, encuadre, tamaño del plano, movimiento, etc. "Les « unités traumatiques » au cinéma", escrito junto a Gilbert Cohen-Séat, desea establecer la metodología científica de la filmología, para definir las unidades significantes análogas de la cadena semántica, siguiendo la semántica estructural de Saussure. Para ello, utilizan los tests filmiques thématiques, donde a una misma secuencia se le aplican diferentes variaciones a fin de analizar cómo cambia la percepción de la misma por parte del espectador. En este caso estudian la duración de una mirada, estableciendo nuevamente una semiología del interior de la imagen, de la comunicación no verbal, que margina una vez más los elementos propiamente cinematográficos.

Sin embargo, Barthes es capaz de detectar las innovaciones fílmicas, como ocurre con el surgimiento del cinéma-verité. En el prefacio escrito para Les Inconnus de la terre, Barthes expone las capacidades de este nuevo realismo: "devant nous, une conscience de classe s'éveille et se parle [...] une clarté circule à travers les images [...] nous ne sentons ici aucun spectacle [...] nous regardons ces images de vérité avec confiance, plaisir et profit"'22. Una capacidad de mostración de la realidad que detecta igualmente a propósito de Chronique d'un été: “C'est le premier film qui me fait voir les autres" "23. Si bien Ffrench relaciona este elogio al realismo como parte de su crítica y resistencia a la ficción cinematográfica y confirmación de la vertiente fotográfica del cine: “the broad thrust of these evaluations on Barthes' part moves toward a critical resistance to the fictional, narrative element in film, a resistance to Pavlovian subjection to the 'story,' and an affirmative evaluation of the element of the photographic capture of the real" 24 , creemos que dichas apreciaciones confirman la capacidad de Barthes para evidenciar las prácticas fílmicas que se alejan de un cine de derechas asociado al cine clásico, y su imagen-acción de la invisibilidad narrativa, y logran generar imágenes de una modernidad de izquierdas que subvierte

\footnotetext{
Watts, P., Le cinéma de Roland Barthes. Suivi d'un entretien avec Jacques Rancière, op.cit., p.181.

Ibidem, p. 77.

Ffrench, P., Roland Barthes and Film. Myth, Eroticism and Poetics, op. cit., pp. 52-53.
} 
el paradigma del clasicismo cinematográfico.

En la entrevista concedida a Cahiers du cinéma, "Sur le cinéma", Barthes reconoce su incapacidad para analizar el cine como un lenguaje: "Pour moi, c'est probablement parce que je n'ai pas réussi à intégrer le cinéma dans la sphère du langage que je le consomme selon un mode purement projectif, et non pas en analyse" 25 . Expone a continuación la que debería ser una semiótica del cine (basándose en la metodología de la filmología), donde encontramos, como ya hemos indicado, una descripción del estudio que años después realizará Christian Metz con sus Essais sur la signification au cinéma $(1968,1973)$ que Barthes alabará en su artículo "Apprendre et enseigner" (1975). Se trata por tanto de un anuncio de una semiótica cinematográfica donde la sintaxis fílmica tomaría el protagonismo:

Je crois que, ce qui serait intéressant à faire, ce serait de voir si un procédé cinématographique peut être converti méthodologiquement en unités signifiantes ; si les procédés d'élaboration correspondent à des unités de lecture du film [...] Le lien du signifiant au signifié a beaucoup moins d'importance que l'organisation des signifiants entre eux [...] Une « bonne histoire », c'est en effet, en termes structuraux, une série réussite de dispatchings syntagmatiques ; étant donné telle situation (tel signe), de quoi peut-elle être suivie ? [...] Tout ceci semble prouver qu'il y a des possibilités d'échange entre la linguistique et le cinéma, à condition de choisir une linguistique du syntagme plutôt qu'une linguistique du signe [...] Comment le cinéma manifeste-t-il ou rejoint-il les catégories, les fonctions, la structure de l'intelligible élaborées par notre histoire, notre société ? C'est à cette question que pourrait répondre une « sémiologie » du cinéma. ${ }^{26}$

Sin embargo, preguntado por la significación política de un film, Barthes expone la capacidad del cine para materializar lo que él denomina la técnica del sentido suspendido:

Je crois que le cinéma a du mal à donner des sens clairs et qu'en l'état actuel il ne doit pas le faire. Les meilleurs films (pour moi) sont ceux qui suspendent le mieux le sens. Suspendre le sens est une opération extrêmement difficile, exigeant à la fois une très grande technique et une loyauté intellectuelle totale. ${ }^{27}$

Por tanto, la función social del cine residiría en una suspensión del sentido que evitaría los dogmatismos y adoctrinamientos, para hacer reflexionar al espectador y desarrollar así su pensamiento crítico. Al solicitarle ejemplos de este sentido suspendido, Barthes cita El ángel exterminador de Buñuel:

on peut voir comment, à chaque moment, le sens est suspendu, sans être jamais, bien entendu, un non-sens. Ce n'est pas du tout un film absurde ; c'est un film qui est plein de sens ; plein de ce que Lacan appelle la « signifiance ». Il est plein de signifiance, mais il n'a pas un sens, ni une série de petits sens. Et par là même, c'est un film qui secoue profondément, et qui secoue au-delà du dogmatisme, au-delà des doctrines. Normalement, si la société des consommateurs de films était moins aliénée, ce film devrait, comme on

25 Barthes, R., Le Grain de la voix : entretiens, 1962-1980, op. cit., p. 20.

Ibidem, pp. 22-25.

Ibidem, pp. 26-27. 
dit vulgairement et justement, « faire réfléchir ». On pourrait d'ailleurs montrer, mais il y faudrait du temps, comment les sens qui « prennent » à chaque instant, malgré nous, sont saisis dans un dispatching extrêmement dynamique, extrêmement intelligent, vers un sens suivant, qui lui-même n'est jamais définitif. ${ }^{28}$

De este modo, Barthes identifica la suspensión del sentido con el concepto de significancia definido por Lacan: "c'est ce qui a effet de signifié" 29 . Es decir, la articulación entre significantes que producirán efectos de significado. Una significancia cuya materialización se produciría en la modernidad cinematográfica representada aquí por Buñuel y que podría residir en una sintagmática cinematográfica, en la elección y combinación de los elementos fílmicos. Una suspensión del sentido que conecta claramente con la imagen-tiempo de Gilles Deleuze que describe igualmente la ruptura generada por la modernidad cinematográfica. La suspensión del sentido barthesiana coincide con la fractura de la cadena causa-efecto que define la imagen-movimiento deleuziana y que da paso a la imagen-tiempo. Esta materializa la indiscernibilidad definida por el autor, que se relaciona igualmente con la suspensión del sentido. Un cine moderno que, como indica Ffrench, ${ }^{30}$ vincula la pensivité barthesiana -que nuevamente negará el cine en La Chambre claire (casi dos décadas después) para reducirla a la imagen detenida ${ }^{31}$ - con la reflexividad que para Deleuze le es definitoria: "L'image cinématographique doit avoir un effet de choc sur la pensée, et forcer la pensée à se penser elle-même comme à penser le tout. C'est la définition même du sublime". ${ }^{32}$

Continúa Barthes en la entrevista: "Si le cinéma moderne a une direction, c'est dans l'Ange exterminateur qu'on peut la trouver [...] les scènes (les fragments syntagmatiques) ne constituent pas une suite immobile (obsessionnelle, métaphorique), elles participent chacune à la transformation progressive d'une société de fête en société de contrainte, elles construisent une durée irréversible"33. Precisamente, Deleuze sitúa esta obra de Buñuel en el pasaje del cineasta hacia la imagen-tiempo:

Soumettre l'image à une puissance de répétition-variation, c'était déjà l'apport de Buñuel, et une façon de libérer le temps, de renverser sa subordination au mouvement. Seulement, dans la plus grande partie de l'œuvre de Buñuel, nous avons vu que le temps restait un temps cyclique, où tantôt l'oubli (Susana), tantôt l'exacte répétition (L'ange exterminateur) marquaient la fin d'un cycle et le début possible d'un autre, dans un cosmos encore unique. ${ }^{34}$

Barthes identifica así la suspensión del sentido con una obra que destruye la cadena causal de la imagen-movimiento al generar una suspensión de la temporalidad. Es decir, se asocia la suspensión del sentido con la capacidad del cine para suspender la temporalidad sin detener la imagen (Imagen 1).

\footnotetext{
Ibidem, p. 27.

Lacan, J., Le Séminaire. Livre XX. Encore: 1972-1973, Paris, Édtition du Seuil, 1975, p. 23.

Ffrench, P., Roland Barthes and Film. Myth, Eroticism and Poetics, op. cit., p. 115.

Barthes, R., Barthes, R., La Chambre claire, op. cit., p. 90.

Deleuze, G., L'Image-temps, Paris, Éditions de Minuit, 1985, p. 206.

Barthes, R., Le Grain de la voix : entretiens, 1962-1980, op. cit., p. 28.

Deleuze, G., L'Image-temps, op.cit., p. 206.
} 


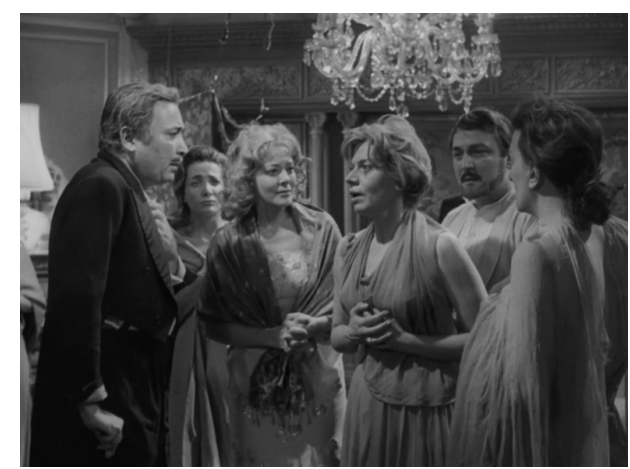

Imagen 1. El ángel exterminador (Luis Buñuel, 1962)

En "Sémiologie et cinéma" (1964) Barthes continúa con la descripción de esa futura semiótica cinematográfica, de nuevo identificada con el análisis sintagmático del estructuralismo, frente a la retórica del eje paradigmático:

On peut, d'une part, chercher à établir la rhétorique du film, c'est-à-dire un inventaire de signes discontinus, les connotateurs [...] Mais, d'autre part, il y a une autre direction de travail qui consiste à reconstituer la structure des récits, ce que Souriau appelait la « diégèse » $[\ldots]$ Et ces deux types d'analyse, bien que faisant partie du même complexe, ne se confondent pas. Cette analyse fonctionnelle est peut-être plus importante, plus riche, plus urgente que l'analyse rhétorique. Dans cette optique, on devine à peu près comment est fait un film, du point de vue opératoire : c'est une sorte de « dispatching », de réseau distributionnel de situations et d'actions. ${ }^{35}$

En ese plano sintagmático y semiótico, Barthes cita de nuevo a un autor de la modernidad como ejemplo de la ruptura con los antiguos códigos que esta implica: "chez Bergman [Le Silence], la rhétorique, en tant qu'ensemble de signes stéréotypés, est sans cesse combattue, déviée, détruite, d'ailleurs souvent au profit d'une autre rhétorique beaucoup plus individuelle et subtile. On peut donc penser dès maintenant que l'analyse sémiologique débouchera un jour sur une esthétique" ${ }^{36}$. Es preciso señalar que Ingmar Bergman genera El silencio desde una cierta suspensión de la coordenada espacio-temporal, de nuevo asociada a una suerte de encierro de los personajes protagonistas (Imagen 2). 


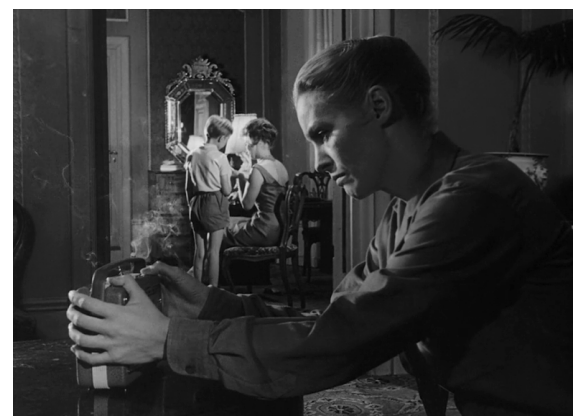

Imagen 2. El silencio (Ingmar Bergman, 1963)

En el Festival de Cine de Pessaro de 1966, Barthes sintetiza nuevamente el reto de un cine moderno que se oponga al realismo burgués asimilado por el cine clásico:

For purely historical and contingent reasons, since its origins the cinema has been linked to a society in which signs are not manifest, a society of shameful signs. How can cinema free itself from the society in which it was born? How it can solve this problem of manifest or shameful signs? How it can come out of the naturalistic model of bourgeois art? How it can pass today to an order of intentionally arbitrary signs? In a word: how can it become an art? I would like to conclude with these questions, while affirming that the task of dealienating the sign by manifesting it, manifesting the code of which it is part, seems to me today to be the real political task, I insist on the word, of every new art. ${ }^{37}$

Barthes cita entonces L'Année dernière à Marienbad como ejemplo de ese nuevo cine del eje sintagmático. Se trata, nuevamente, de una obra clave de la modernidad cinematográfica y de la imagen-tiempo que Deleuze analiza en profundidad como materialización paradigmática de esta: "une dissolution générale de l'imageaction" 38 . Si el film de Buñuel generaba la suspensión del sentido a través de la idea de repetición temporal, el film de Resnais surge de la simultaneidad de temporalidades:

Il n'y a jamais chez lui succession des présents qui passent, mais simultanéité d'un présent de passé, d'un présent de présent, d'un présent de futur, qui rendent le temps terrible, inexplicable [...] les trois présents impliqués se reprennent toujours, se démentent, s'effacent, se substituent, se recréent, bifurquent et reviennent. C'est une image-temps puissante $[. .$.$] elle donne à la narration une nouvelle valeur, puisqu'elle l'abstrait de toute$ action successive, pour autant qu'elle substitue une véritable image-temps à l'imagemouvement. ${ }^{39}$

Una imagen-tiempo que, una vez más, alcanza la indiscernibilidad:

Nous avons vu que, dans l'image, la distinction se fait toujours entre le réel et l'imaginaire, l'objectif et le subjectif, le physique et le mental, l'actuel et le virtuel, mais que cette distinction devient réversible, et en ce sens indiscernable. Distincts et pourtant

37 Ffrench, P., Roland Barthes and Film. Myth, Eroticism and Poetics, op. cit., pp. 93-94.

38 Deleuze, G., L'Image-temps, op.cit., p. 136.

39 Ibidem, p. 133. 
indiscernables, tels sont l'imaginaire et le réel chez l'un et l'autre des deux auteurs [...] Ce n'est plus, ou ce n'est plus seulement le devenir indiscernable d'images distinctes, ce sont des alternatives indécidables entre des cercles de passé, des différences inextricables entre des pointes de présent. ${ }^{40}$

Se confirma entonces el vínculo entre el sentido suspendido barthesiano y la suspensión cinematográfica de la temporalidad. Si los protagonistas de El ángel exterminador quedaban presos en una suspensión temporal que solo se resolvía con la repetición, el film de Resnais genera esa misma suspensión temporal a partir de la memoria de sus personajes (Imagen 3). Es decir, ambos films logran suspender la temporalidad sin detener la imagen. La película de Bergman, sin embargo, genera la suspensión del sentido a partir de dos elementos que aparecen a continuación en el análisis de Barthes, a partir de los escritos sobre Pasolini y Antonioni: la idea del encierro y el aislamiento (también en Buñuel) y la noción de vaciamiento y extrañamiento.

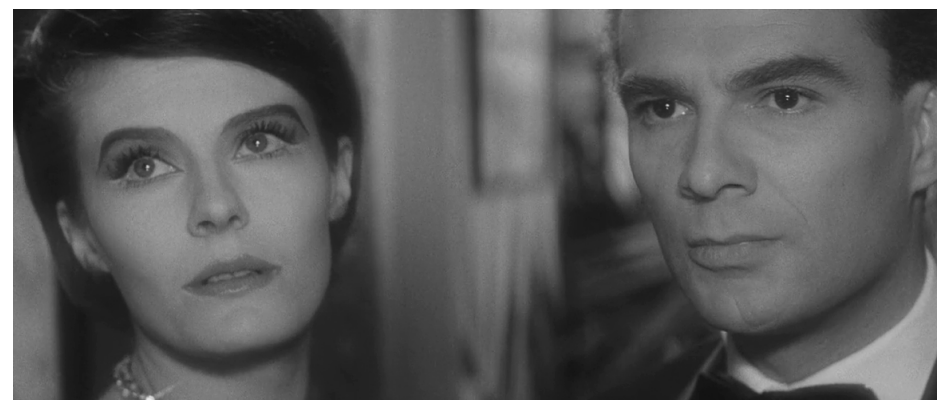

Imagen 3. L'Année dernière à Marienbad (Alain Resnais, 1961)

\section{El sentido obtuso}

Ya en 1970, con la publicación de "Le troisième sens", el artículo a propósito del cine de Eisenstein, se produce la paradoja por la cual le sens obtus, el que hasta ahora Barthes había denominado sentido suspendido, escapa de la semiótica para materializar la contradicción de esa resistencia cinematográfica. Al tiempo que define lo fílmico como la esencia de un cine que responde a la práctica de la modernidad, y que se relaciona con el post-estructuralismo, reduce el mismo al fotograma, a la imagen detenida. En el artículo que Pascal Bonitzer, alumno de Barthes, le dedica en Cahiers $d u$ cinéma tras su muerte, titulado "Le hors-champ subtil" -término utilizado por Barthes en La Chambre claire- escribe: "Il résistait donc et le noyau de cette résistance était ce qui, d'après les spécialistes, constitue l'essence du cinéma, à savoir le mouvement [...] Ce que Barthes aimait dans le cinéma, pourrait-on croire, c'est donc la photographie, ou si l'on veut le photogramme. Ce n'est pas le cinéma"41. Por tanto, desaparece así la aproximación semiótica del eje sintagmático y Barthes desarrolla la noción de suspensión de sentido para definir el sentido obtuso, que identifica nuevamente como la significancia, relacionada ahora con el uso que 
del término hace Julia Kristeva en su semiótica del texto (1969), y conectando así con las teorías post-estructuralistas. El sentido obtuso es una y otra vez descrito a partir del análisis de los films de Eisenstein: "une emphase elliptique"; "une feuilleté de sens qui laisse toujours subsister le sens précédent, comme dans une construction géologique ; dire le contraire sans renoncer à la chose contredite"; "le sens obtus porte une certaine émotion [...] c'est une émotion-valeur, une évaluation" ${ }^{42}$. Un sentido obtuso que, si en la entrevista con Cahiers podría ser pieza clave de una sintagmática cinematográfica, Barthes ahora lo expulsa del ámbito semiótico: "le sens obtus n'est pas situé structuralement, un sémantologue ne conviendra pas de son existence objective", "le sens obtus et un signifiant sans signifié ; d'où la difficulté a le nommer ; ma lecture reste suspendue entre l'image et sa description, entre la définition et l'approximation", "le sens obtus ne parviendra pas à exister, à entrer dans le métalangage du critique [...] ce que le sens obtus trouble, stérilise, c'est le métalangage (la critique)"43. Es entonces cuando el sentido obtuso, la suspensión del sentido, expulsado de la esfera de una semiótica del cine, se define mediante una esencia fílmica, le filmique, que, paradójicamente, reside en lo fotográfico:

Le filmique, c'est, dans le film, ce qui ne peut être décrit, c'est la représentation qui ne peut être représentée. Le filmique commence seulement là où cessent le langage et le métalangage $[\ldots]$ le filmique est donc exactement là, dans ce lieu où le langage articulé n'est plus qu'approximatif et où commence un autre langage $[\ldots]$ Le troisième sens, que l'on peut situer théoriquement mais non décrire, apparait alors comme le passage du langage à la signifiance et l'acte fondateur du filmique même. [...] Car le filmique est différent du film : le filmique est aussi loin du film que le romanesque du roman (je puis écrire du romanesque, sans jamais écrire de romans [...] C'est pourquoi, dans une certaine mesure (qui est celle de nos balbutiements théoriques), le filmique, très paradoxalement, ne peut être saisi dans le film « en situation », « en mouvement », « au naturel », amis seulement, encore, dans cet artefact majeur qu'est le photogramme [...] le mouvement dont on fait l'essence du film n'est nullement animation, flux, mobilité. «vie », copie, mais seulement l'armature d'un déploiement permutatif, et une théorie du photogramme est nécessaire. ${ }^{44}$

La primera evidencia es que el sentido obtuso del fotograma es una anticipación del punctum fotográfico, sobre el que escribirá el autor una década después. Ya hallamos aquí expuestas, por tanto, las ideas clave de La Chambre claire, como afirma Bonitzer: "il y a le même rapport entre le studium et le punctum dans l'espace photographique, qu'entre le sens obvie et le sens obtus dans l'espace parafilmique du photogramme. Le sens obtus, comme le punctum, relève d'une revendication fétichiste du spectateur" ${ }^{45}$. El rechazo a la imagen en movimiento es la consecuencia directa de la fetichización de la imagen, que debe detener su movimiento, y por tanto su temporalidad, desde el exterior del desarrollo cinematográfico, como también analiza Didi-Huberman:

\footnotetext{
42 Barthes, R., L'Obvie et l'obtus, Paris, Éditions du Seuil, 1982, pp. 49, 51.

43 Ibidem, pp. 54,55.

44 Ibidem, pp. 58-60.

45 Bonitzer, P., "Le hors-champ subtil", op. cit. p. 5.
} 
C'est, premièrement, le fétichisme qui immobilise toutes les surfaces du réel dans un cadre où le réel devient pur tableau [...] un fétichisme qui est arrêt sur l'image, arrêt du sens dans l'image, arrêt de mort de sa libre inventivité [...] il se sent prisonnier du cadre (pervers) comme de l'agir (hystérique) qui lui sont imposés au cinéma. ${ }^{46}$

Sin embargo, no consideramos que esta provenga de la relación del espectador con la imagen, como indica Bonitzer, sino de la que mantiene, de forma intima, el propio Barthes:

Le « sens obtus », donc, subvertit la signification dans l'image [...] Le « sens obtus » déjoue l'histoire qui tend à se raconter dans l'image [...] le sens obtus est un signifiant sans signifié ; d'où la difficulté à le nommer : ma lecture reste suspendue entre l'image et sa description, entre définition et l'approximation [...] Il est donc impartageable, c'est pourquoi il me divise de la communauté des lecteurs, des locuteurs. ${ }^{47}$

De este modo, Barthes crea y destruye el sentido suspendido de la imagen cinematográfica en tres textos. Más concretamente, lo crea en dos entrevistas y lo destruye en el único artículo de análisis cinematográfico en el que lo estudia. Es decir, en los artículos teóricos y en las entrevistas, Barthes es capaz de describir los parámetros y las vías de desarrollo de una semiótica de la imagen cinematográfica, al tiempo que enuncia la noción de suspensión del sentido como el reto de la misma, materializado en las experiencias fílmicas de la modernidad cinematográfica. Sin embargo, cuando se enfrenta personalmente a la imagen fílmica, su escritura autobiográfica, acto de fetichización, niega la imagen cinematográfica, ya que su movimiento no le permite fetichizarla. Afirma Rancière: "lorsque Barthes parle du cinéma, il ne parle à aucun moment du cinéma ! Avec les « photogrammes » d'Eisenstein, il est devant des images, et il n'est pas dans une salle de cinéma ; lorsqu'il est dans une salle de cinéma, il ne parle absolument pas de ce qui se passe sur 1'écran" 48 . Del mismo modo, Raymond Bellour, también alumno de Barthes, señala esta paradoja: “C' $\mathrm{C}$ 'st dire à quel point Barthes vise ici un objet paradoxal : un sens antérieur à toute signification, irréductible au langage articulé, mais cependant porté par lui. Il laisse ainsi ouverte la contradiction qui lui fait d'abord trouver le troisième sens hors du film pour ensuite l'y exhumer de l'intérieur" ${ }^{49}$. Así, aquella resistencia barthesiana se convierte en negación. Lo fílmico no existe, porque es reducido a lo fotográfico. No es posible para Barthes escribir sobre el arte cinematográfico sin negarlo: "his ambivalent denial of any potential for 'pensiveness' in the moving image. It pushes film theory into the impossible situation of positing its object outside the moving image itself, deriving its impetus from the apparent aporia: the film is not the film" 50 . Es por ello que sus mayores aportaciones sobre el mismo se hayan en las entrevistas analizadas y en la carta-discurso redactada con ocasión de un homenaje a Antonioni (1980), que abordaremos a continuación.

En "Diderot, Bretch, Eisenstein" (1973), el instante preñado de G. E. Lessing (1766) yace nuevamente en un gesto captado e inmovilizado por el fotograma a partir

\footnotetext{
Didi-Huberman, G., Peuples en larmes, peuples en armes. L'oeil de l'Histoire, 6, op. cit., pp. 103-105. Ibidem, pp.112-114.

Watts, P., Le cinéma de Roland Barthes. Suivi d'un entretien avec Jacques Rancière, op.cit., p.168. Bellour, R., L'entre-images. Cinéma. Vidéo. Photo, Paris, La Différence, 2002, p. 115.

Ffrench, P., Roland Barthes and Film. Myth, Eroticism and Poetics, op. cit., p. 125.
} 
del que construir esa semiótica de la comunicación no verbal que nada tiene que ver con la práctica cinematográfica. Como indica Rancière: "C'est qui intéresse Barthes, c'est le geste" 51 , quien detecta ese mismo fetichismo en el artículo "En sortant du cinéma" (1975), publicado en el número 23 de la revista Communications, dedicado a Cine y psicoanálisis. Fetichismo que, por definición, niega lo cinematográfico: 'Lorsqu'il revendique un certain fétichisme, c'est un fétichisme qui ne peut que s'opposer à ce qu'est le cinéma comme art des images en mouvement. Le fétichisme, en effet, pour Roland Barthes, c'est la possibilité de toujours découper son petit bout de film, son petit fragment" ${ }^{\prime 2}$.

En este período, destaca la elección de un nuevo film clave de la modernidad cinematográfica, Salò o le 120 giornate di Sodoma (Pier Paolo Pasolini, 1975), que sin embargo Barthes ya no aborda desde la idea anterior de la suspensión del sentido, sino, bien al contrario, desde la idea de la fidelidad al texto literario de Sade: "Pasolini a filmé ses scènes à la lettre", que tendría como resultado el despojamiento de la mirada del espectador:

À ce point de rigueur, ce n'est finalement pas le monde peint par Pasolini qui est dénudé, c'est notre regard : notre regard mis à nu, tel est l'effet de la lettre. Dans le film de Pasolini (ceci, je crois, lui appartenait en propre) il n'y a aucun symbolisme : d'un côté une grossière analogie (le fascisme, le sadisme), de l'autre la lettre, minutieuse, insistante, étalée, léchée comme une peinture de primitif ; l'allégorie et la lettre, mais jamais le symbole, la métaphore, l'interprétation. ${ }^{53}$

Es decir, Barthes retoma la temática de la representación, y sus consecuencias sobre el espectador, y abandona la construcción sintagmática, obviando igualmente la idea de encierro que conecta el film con los anteriormente analizados de Buñuel, Bergman y Resnais, en relación a la suspensión del sentido. Por el contrario, Deleuze sitúa el film bajo el mismo paradigma que L'Année dernière à Marienbad: "la rupture du lien sensori-moteur (image-action), et plus profondément du lien de l'homme et du monde (grande composition organique)" "54. Además, erige Salò como cumbre del cine de pensamiento, que hace: "suivre à la pensée les chemins de sa propre nécessité, et porter l'image au point où elle devient déductive et automatique, substituer les enchaînements formels de la pensée aux enchaînements représentatifs ou figuratifs sensori-moteurs" 55 . Y también subraya la idea de encierro que, como ya hemos señalado, acercaría el film al sentido suspendido barthesiano de la etapa anterior: "Pasolini met en scène, non pas même le fascisme in vivo, mais le fascisme aux abois, renfermé dans la petite ville, réduit à une intériorité pure, coïncidant avec les conditions de fermeture où se déroulaient les démonstrations de Sade. Salo est un pur théorème mort, un théorème de la mort" 56 (Imagen 4).

\footnotetext{
Watts, P., Le cinéma de Roland Barthes. Suivi d'un entretien avec Jacques Rancière, op.cit., p. 164Ibidem, pp. 168-169.

Barthes. R., Euvres Complètes Tome IV, 1968-1976, Paris, Éditions du Seuil, 2002, p. 944

Deleuze, G., L'Image-temps, op.cit., p. 225.

Ibidem, p. 226

Ibidem, p. 228.
} 


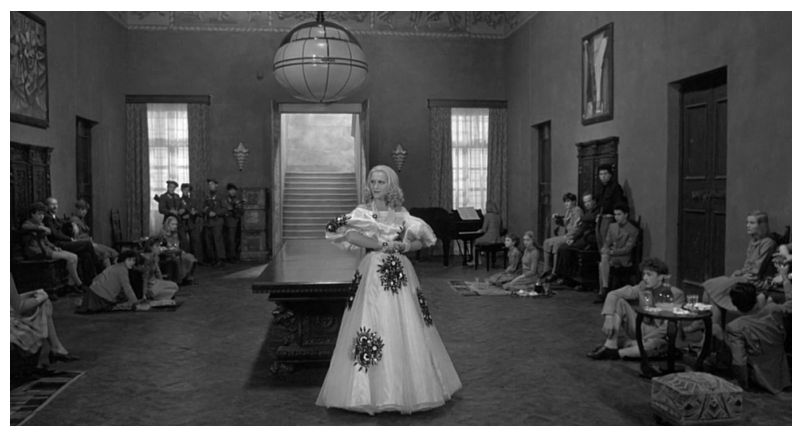

Imagen 4. Salò o le 120 giornate di Sodoma (Pier Paolo Pasolini, 1975)

\section{Michelangelo Antonioni}

En "Cher Antonioni" (1980), carta abierta dirigida al cineasta con motivo de la entrega de un premio en Bolonia en enero de 1980, Barthes realiza una espléndida disertación sobre la noción de artista, personificada en Antonioni. Sus esencias: vigilance, sagesse, fragilité, van describiendo de manera lúcida al cineasta de una modernidad que también define:

le Moderne est bien au contraire une difficulté active à suivre les changements du Temps, non plus seulement au niveau de la grande Histoire, mais à l'intérieur de cette petite histoire dont l'existence de chacun de nous est la mesure [...] chacun de vos films a été, à votre propre échelle, une expérience historique, c'est-à-dire l'abandon d'un ancien problème et la formulation d'une nouvelle question. ${ }^{57}$

Artista de la modernidad al que le atribuye igualmente la capacidad de suspender el sentido:

vous témoignez d'un sentiment juste du sens : vous ne l'imposez pas, mais vous ne l'abolissez pas. Cette dialectique donne à vos films une grande subtilité : votre art consiste toujours à laisser la route du sens ouverte, et comme indécise, par scrupule. C'est en quoi vous accomplissez très précisément la tâche de l'artiste dont notre temps a besoin : ni dogmatique ni insignifiant. ${ }^{58}$

Barthes va citando los films de Antonioni -Il grido, L'eclisse, Il deserto rosso (Imagen 5) - para ilustrar de forma sintética la práctica de la modernidad cinematográfica, hasta enunciar la especificidad de su obra:

$\mathrm{Au}$ fond, au fil de votre œuvre, il y a une critique constante, à la fois douloureuse et exigeante, de cette marque forte du sens, qu'on appelle le destin. Cette vacillation - j'aimerais mieux dire avec plus de précision : cette syncope du sens, suit des voies techniques, proprement filmiques (décor, plans, montage) qu'il ne m'appartient pas d'analyser, car je n'en ai pas la compétence. ${ }^{59}$

57 Barthes, R., Euvres Complètes Tome V, 1977-1980, Paris, Éditions du Seuil, 2002, p. 900.

$58 \quad$ Ibidem, p. 901.

$59 \quad$ Ibidem, p. 902. 


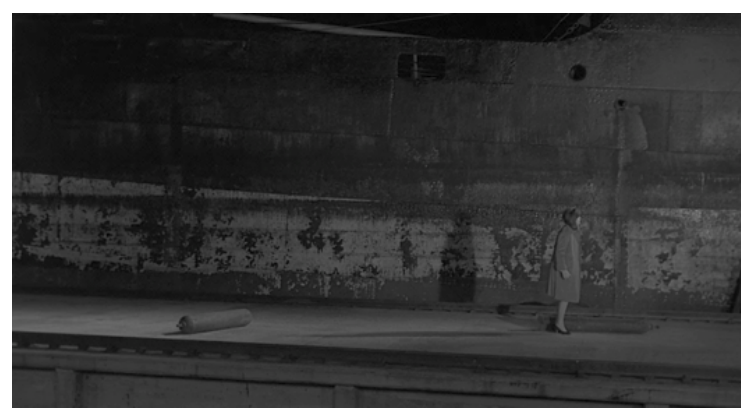

Imagen 5. Il deserto rosso (Michelangelo Antonioni, 1964)

Barthes, de nuevo, y en contradicción con lo afirmado en "Le troisième sens", relaciona esa síncopa del sentido con la técnica cinematográfica-planos, montaje-a cuyo análisis él no puede acceder. De este modo, la suspensión del sentido como esencia de la modernidad cinematográfica estaría relacionada de nuevo con una semiótica fílmica que Barthes así anuncia. Reaparece entonces la conexión con Deleuze en el uso del término intersticio: "D'une certaine manière, votre art est lui aussi un art de l'interstice (de cette proposition, L'Avventura serait la démonstration éclatante)" ${ }^{" 60}$. Un intersticio desarrollado ampliamente por Deleuze, y que este sí que sitúa fílmicamente en el espacio entre imágenes:

L'image moderne instaure le règne des " incommensurables " ou des coupures irrationnelles : c'est-à-dire que la coupure ne fait plus partie de l'une ou l'autre image, de l'une ou l'autre suite qu'elle sépare et répartit [...] L'intervalle se libère, l'interstice devient irréductible et vaut pour lui-même. La première conséquence est que les images ne s'enchaînent plus par coupures rationnelles, mais se ré-enchaînent sur coupures irrationnelles. ${ }^{61}$

Frente a la suspensión del sentido a través de la temporalidad de los films de Buñuel y Resnais, Deleuze señala la exploración de los tiempos muertos y los espacios vacíos en Antonioni, lo que nos remite también a la película de Bergman:

Et l'art d'Antonioni ne cessera de se développer dans deux directions : une étonnante exploitation des temps morts de la banalité quotidienne ; puis, à partir de L'Éclipse, un traitement des situations-limites qui les pousse jusqu'à des paysages déshumanisés, des espaces vidés dont on dirait qu'ils ont absorbé les personnages et les actions, pour n'en garder qu'une description géophysique, un inventaire abstrait. ${ }^{62}$

De este modo, las imágenes se convierten en mentales; categorización con la que podríamos vincular las películas citadas: "chez Antonioni, on dirait que les images les plus objectives ne se composent pas sans devenir mentales, et passer dans une étrange subjectivité invisible", a través de las cuales se alcanza nuevamente la suspensión de la temporalidad, y del sentido, que detecta Barthes: "on remarque que

\footnotetext{
$60 \quad$ Ibidem, p. 902.

61 Deleuze, G., L'Image-temps, op.cit., p. 362.

62 Ibidem, p. 12
} 
les images objectives d'Antonioni, qui suivent impersonnellement un devenir, c'està-dire un développement de conséquences dans un récit, n’en subissent pas moins de rapides ruptures, intercalages, « infinitésimales injections d'atemporalité »" "63. Esta atemporalidad del cine del cerebro se hibrida con la fatiga de los personajes, cine del cuerpo, que también caracterizaban las películas citadas de Buñuel, Bergman y Resnais:

Si bien que son œuvre passe fondamentalement par un dualisme qui correspond aux deux aspects de l'image-temps : un cinéma du corps, qui met tout le poids du passé dans le corps, toutes les fatigues du monde et la névrose moderne ; mais aussi un cinéma du cerveau, qui découvre la créativité du monde, ses couleurs suscitées par un nouvel espace-temps, ses puissances multipliées par les cerveaux artificiels. ${ }^{64}$

Continuando con el texto de Barthes, reaparece entonces el vínculo entre el sens obtus y la concepción política de la actividad artística que se trataba en "Le troisième sens", pero esta vez sin negar el hecho cinematográfico, y enunciando también la dialéctica espacio-tiempo que será crucial en Deleuze: "Subtiliser le sens est donc une activité politique seconde, comme l'est tout effort qui vise à effriter, à troubler, à défaire le fanatisme du sens [...] voyageur einsteinien, il [l'artiste] ne sait jamais si c'est le train ou l'espace-temps qui bouge, s'il est témoin ou homme de désir"'65. Barthes concluye su carta con una síntesis de su concepción del artista:

car être artiste aujourd'hui, c'est là une situation qui n'est plus soutenue par la belle conscience d'une grande fonction sacrée ou sociale [...] c'est, au moment de chaque œuvre, devoir affronter en soi ces spectres de la subjectivité moderne, que sont [...] Ia lassitude idéologique, la mauvaise conscience sociale, l'attrait et le dégoût de l'art facile, le tremblement de la responsabilité, l'incessant scrupule qui écartèle l'artiste entre la solitude et la grégarité. ${ }^{66}$

Comprobamos nuevamente, cómo, cuando la expresión de Barthes no es escritura autobiográfica, no es expresión íntima de su relación con el cine, el autor puede entonces exponer sus argumentaciones más fértiles sobre el arte cinematográfico, en este caso en relación a Antonioni como personificación de ese cine de la suspensión del sentido. Una noción que, de acuerdo con Bonitzer y su hors-champ subtil desvela la esencia del cine de la Modernidad:

Le hors-champ subtil apparaît dans la photographie avec le punctum, dans le photogramme ou la photo du film avec le sens obtus. Seul le cinéma moderne double ou refend le hors-champ étoffé d'un hors-champs subtil, en appelle à la place du spectateur, à son énonciation. Mais cette place, quelle est-elle ? [...] cette place est sur l'écran, dans une soudaine opacité ou étrangeté de la scène [...] Il se crée alors un vacillement de la représentation, un vertige, une béance. ${ }^{67}$

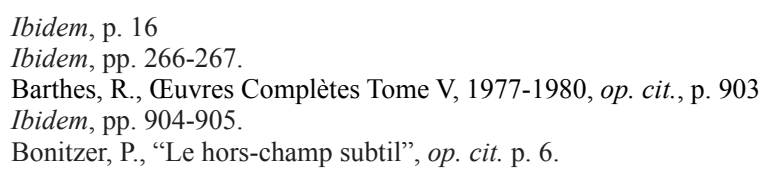


Watts, por su parte, al analizar la misiva, destaca la relación entre la suspensión del sentido y la insistencia de la mirada, como fórmula de la reflexión asociada a la modernidad, que ya hemos mencionado anteriormente respecto a los films de Buñuel y de Pasolini:

Barthes place cette suspension du sens dans une technique cinématographique très précise, ce que Barthes appelle « l'insistance du regard » [...] Barthes insiste sur le fait que les films d'Antonioni sont construits autour d'un regard soutenu sur le monde qui défait tout sens dogmatique [...] Autrement dit, selon Barthes, les films d'Antonioni n'imposent pas un sens ; ils permettent la production de pensées, d'hypothèses, du travail simultané de la perception et de l'imagination, de ce qu'il appelle la pensitivé. ${ }^{68}$

\section{Conclusiones}

La resistencia de Barthes frente a la materia cinematográfica reside en la imposibilidad de fetichizar la imagen en movimiento, que debe entonces ser reducida a lo fotográfico. Resistencia que se convierte en negación cuando la escritura barthesiana se hace más íntima y autobiográfica, y que no obstante ha proporcionado textos de gran interés tanto en el ámbito de la crítica marxista y brechtiana del engagement de los años cincuenta, como en la descripción del camino a seguir por una semiótica de la imagen cinematográfica en los sesenta, para revelar la esencia del cine de la modernidad, la suspensión del sentido, en autores tan determinantes como Buñuel, Bergman, Resnais, Pasolini o Antonioni. Una trayectoria que se define por la paradoja barthesiana entre lo fílmico y el cine: “C'est bien là ce que Barthes a toujours reproché au cinéma, en l'opposant à la photo : son hystérie, sa voracité, son défaut de pensivité (c'est là ce qui le renvoyait au plaisir du photogramme). Impossible d'arrêter la machine, et de se vivre en elle"69. Ante esta imposibilidad, Barthes transforma la imagen en movimiento en fotograma, el cine en lo que él denomina lo fílmico. Sin embargo, señala la esencia de la modernidad cinematográfica que él no puede analizar: la capacidad del cine para suspender la temporalidad sin detener la imagen, sin congelar el movimiento. Así, Barthes detecta relevantes materializaciones de lo que Deleuze más tarde denominará la imagen-tiempo. El sentido suspendido se convierte entonces en la máxima aproximación barthesiana de una esencia verdaderamente fílmica, capaz de suspender el sentido sin detener el movimiento. La suspensión de la temporalidad, la destrucción de la imagen-acción de Deleuze, es la verdadera esencia del cinematógrafo que revela Barthes, ya que determina su punto ciego, el límite de su capacidad de análisis fílmico.

\section{Referencias bibliográficas}

Barthes, R., Mythologies, Paris, Éditions du Seuil, 1957.

Barthes, R., La Chambre claire, Paris, Éditions de l'Étoile, Gallimard, Le Seuil, 1980.

Barthes, R., Le Grain de la voix : entretiens, 1962-1980, Paris, Éditions du Seuil, 1981.

\footnotetext{
68 Watts, P., Le cinéma de Roland Barthes. Suivi d'un entretien avec Jacques Rancière, op.cit., p. 97.

69 Bellour, R., L'entre-images. Cinéma. Vidéo. Photo, op. cit., p. 71.
} 
Barthes, R., L'Obvie et l'obtus, Paris, Éditions du Seuil, 1982.

Barthes, R., Euvres Complètes Tome I, 1942-1961, Paris, Éditions du Seuil, 2002.

Barthes, R., Euvres Complètes Tome II, 1962-1967, Paris, Éditions du Seuil, 2002.

Barthes. R., Euvres Complètes Tome IV, 1968-1976, Paris, Éditions du Seuil, 2002.

Barthes, R., Euvres Complètes Tome V, 1977-1980, Paris, Éditions du Seuil, 2002.

Bellour, R., L’Entre-images. Cinéma. Vidéo. Photo, Paris, La Différence, 2002.

Bonitzer, P., "Le hors-champ subtil", Cahiers du cinéma, 311, 1980. pp. 4-7.

Deleuze, G., L'Image-temps, Paris, Éditions de Minuit, 1985.

Didi-Huberman, G., Peuples en larmes, peuples en armes. L'oeil de l'Histoire, 6, Paris, Éditions de Minuit, 2016.

Ffrench, P., Roland Barthes and Film. Myth, Eroticism and Poetics, London, Bloomsbury Academic, 2020.

Kristeva, J., Semeiotikê. Recherches pour une sémanalyse, Paris, Éditions du Seuil, 1969.

Lacan, J., Le Séminaire. Livre XX. Encore: 1972-1973, Paris, Édtition du Seuil, 1975.

Metz, C., Essais sur la signification au cinema I, Paris, Klincksieck, 1968.

Metz, C., Essais sur la signification au cinema II, Paris, Klincksieck, 1973.

Ricœur, P., Du texte à l'action, Paris, Éditions du Seuil, 1986.

Truffaut, F., "Un certain regard du cinéma français", Cahiers du cinéma, 31, 1954, pp. 15-29.

Watts, P., "Roland Barthes's Cold-War Cinema", SubStance, \#108, 34(3), 2005, pp. 17-32. https://www.jstor.org/stable/3685729

Watts, P., Le cinéma de Roland Barthes. Suivi d'un entretien avec Jacques Rancière, Paris, De l'incidence, 2015. 